\title{
Status of ionization by radial electron neat adaptation ion source research and development for SPIRAL2 and EURISOL-DS ${ }^{\text {a) }}$
}

\author{
C. Lau, M. Cheikh Mhamed, and S. Essabaa \\ IPN Orsay, Division Accélérateurs, Groupe Cibles-Sources d'ions, F-91406 Orsay Cedex, France
}

(Presented 31 August 2007; received 31 August 2007; accepted 18 December 2007; published online 31 January 2008)

To take up the challenging issue of supplying a plasma ion source able to produce radioactive beams under extreme SPIRAL2 and EURISOL irradiation conditions, a research and development program has been initiated to work out ionization by radial electron neat adaptation (IRENA) ion source. Based on the electron beam generated plasma concept, the ion source is specifically adapted for thick target exploitation under intense irradiation. A validation prototype has been designed, constructed, and tested. First results obtained will be presented and commented. IRENA potential will be discussed, particularly in the framework of multimegawatt EURISOL. (C) 2008 American Institute of Physics. [DOI: 10.1063/1.2834316]

\section{INTRODUCTION}

SPIRAL2 (Ref. 1) and EURISOL-DS (Ref. 2) projects aim at producing various neutron-rich-nuclear ion beams as intense as possible, using isotope separation on line (ISOL) techniques with a thick fission target. The characteristics of the beams to be produced depend primarily on the design of the target-ion-source (TIS) system. The irradiation conditions considered in both projects and the need of beam purity for exploitation demand an exhaustive development program to work out a proper design, in particular, for ion source. In spite of significant differences in irradiation conditions between these two projects, there are two common fundamental specifications: reliable operation under strong vapor pressure and under high radiation level.

Strong vapor pressure comes from the need to increase the total surface of the bulk material composing the fission target. Indeed, the fission targets considered for these projects are about an order of magnitude thicker than today's, with a porous structure for fast release characteristics. As pumping in the TIS can only occur through the ion source emission hole, the pressure in the ion source tends to be comparable to the one in the target volume. Indeed, any pumping outlet before the ionization chamber is prohibited to prevent taking away nuclei of interest before they reach the ion source; the small conductance of the ion source emission hole drastically limits the effective pumping speed in the TIS.

High radiation level comes from the need to have the ion source close to the target which is in the vicinity of the neutron converter irradiated by high power primary beam. The neutron flux at ion source location is of the order of $10^{14}$ neutrons $/ \mathrm{cm}^{2} / \mathrm{s}$ for the two projects, with gamma and neutron spectra being of course quite different. Ion source materials, particularly insulators, have to be properly se-

a) Contributed paper, published as part of the Proceedings of the 12th International Conference on Ion Sources, Jeju, Korea, August 2007. lected taking into account existing data. ${ }^{3}$ Furthermore, ion source prototypes have to be designed as simple as possible, minimizing the amount of components to assure highest reliability as well as lowest material activation. The lowest activation possible is also required as a safety specification and for radioactive waste handling. These fundamental specifications demand a multitude of developments and tests to work out an operational ion source, whatever the kind of ion source considered.

\section{RESEARCH AND DEVELOPMENT ON IRENA ION SOURCE}

Currently, no radioactive ion source fulfils all these fundamental operation specifications. The vapor flow from the target is troublesome for all ion sources, e.g., forced electron beam induced arc discharge ${ }^{4}$ (FEBIAD)-type ion sources normally operate with an incoming gas flow below a few $10^{-3} \mathrm{mbar} 1 / \mathrm{s}$ while the vapor flow generated in the target area by the future uranium carbide at $2000^{\circ} \mathrm{C}$ is estimated to exceed $10^{-1} \mathrm{mbar} 1 / \mathrm{s}$. In contrast, Nielsen or Nier-Bernas ion sources can deliver a few milliamperes with an emittance of $20 \pi \mathrm{mm}$ mrad at $40 \mathrm{kV}$ but producing an intense arc discharge in that vapor pressure leads to a quick wearing of the cathode. So it has been decided to establish a research and development $(\mathrm{R} \& \mathrm{D})$ program focused on a FEBIAD-type ion source capable of operating with higher vapour flows. Taking into account all the operation specifications, this program aims at working out a dedicated FEBIAD-type ion source with a cylindrical cathode: ionization by radial electron neat adaptation (IRENA). ${ }^{5,6}$ The first IRENA prototype has been worked out at the ALTO facility; ${ }^{7}$ tests of this feasibility prototype are still in progress.

\section{IRENA FEASIBILITY PROTOTYPE}

The feasibility prototype has been designed for a standard thick target. The dimensions of the ionization chamber are close to those of electron beam generated plasma (EBGP) 


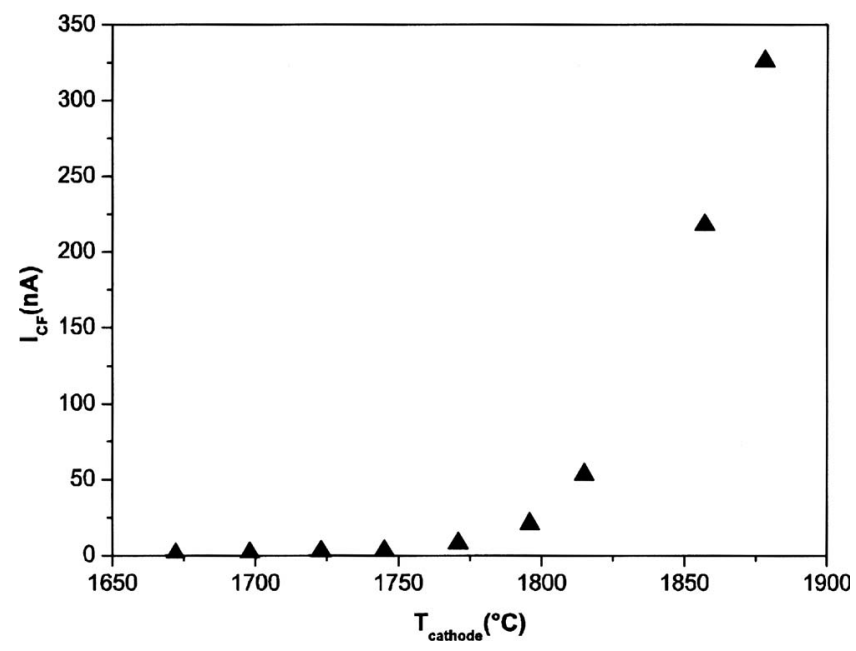

FIG. 1. Evolution of the total ion beam current extracted from IRENA feasibility prototype as function of cathode temperature (Ref. 10). The ion beam current has been measured with a Faraday cup inserted at the focal point before the mass separator. The arc voltage applied is $100 \mathrm{~V}$.

to have a comparison basis with existing measurements. ${ }^{5}$ Contrary to $\mathrm{EBGP}^{8}$, the cathode is Ohmically heated to get reliable operation and to assure a negligible magnetic field inside. The electrical power required to heat a $1 \mathrm{~mm}$ thick Ta cathode to $2000{ }^{\circ} \mathrm{C}$ is about $5 \mathrm{~kW}$. To better understand the effect of the differences between these two ion sources and also to prepare the design of the upgraded IRENA prototype, the feasibility prototype has been modeled using Lorentz codes, see Ref. 9. Figure 1 shows the evolution of the ion beam current extracted as function of the cathode temperature, measured by a Faraday cup inserted at the focal point before the mass separator. These measurements have been performed with arc voltage at $100 \mathrm{~V}$. The ion beam current starts to increase very significantly while the cathode temperature approaches $2000{ }^{\circ} \mathrm{C}$. It has a similar evolution as the electron bombardment current.

Measurements at higher cathode temperature as function of all other ion source parameters are in progress. All the measurements have been carried out with an unfavorable pressure of about $10^{-5}$ mbar at the extraction-electrode vicinity. Because of restricting the dimensions of the ionization chamber, the beam current extracted is weak even when cathode temperature is close to $1900{ }^{\circ} \mathrm{C}$. However, regarding the evolution of Fig. 1, the ion beam current could already microampere intensities with a cathode at around $2000{ }^{\circ}$ C. Such intensities would be comparable to those obtained for EBGP under normal operation conditions. Higher intensities are then expected while looking for optimal operation conditions with a Ta cathode at about $2400{ }^{\circ} \mathrm{C}$.

Figure 2 shows mass spectra of extracted ion beams achieved at three different cathode temperatures: 1770, 1810, and $1860{ }^{\circ} \mathrm{C}$. The evolution of identified masses as function of cathode temperature fits the evolution of the total ion beam current, except for ${ }^{23} \mathrm{Na}$ and ${ }^{39} \mathrm{~K}$. This exception is due to the fact that a part of these alkalis have been produced by surface ionization. This has been checked by measurements without arc voltage.

Considering $\mathrm{Cu}^{+}, \mathrm{Ta}^{++}$, and $\mathrm{TaO}^{++}$beams, observable

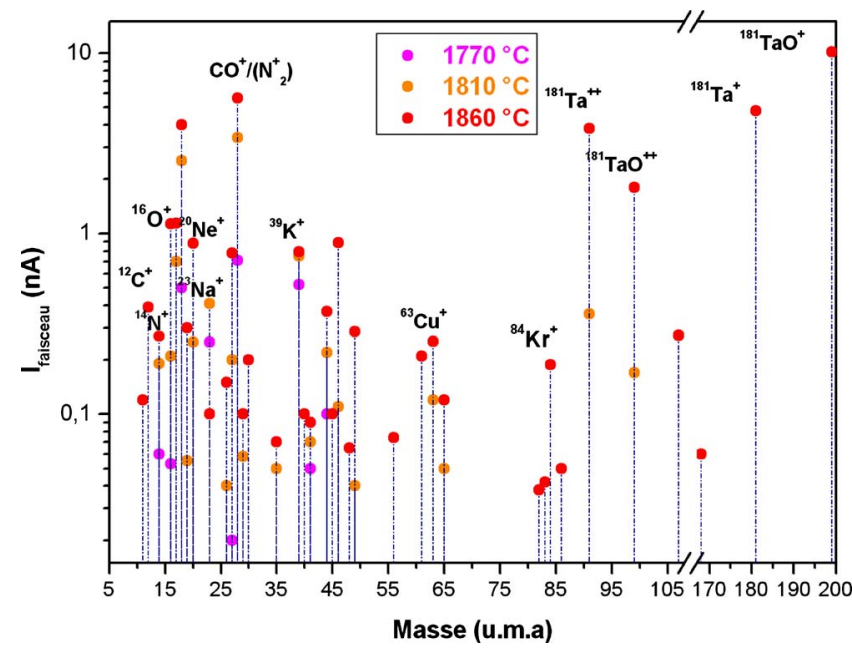

FIG. 2. (Color online) Mass spectrum of beams extracted from IRENA feasibility prototype obtained at the off-line mass separator of ALTO facility; the arc voltage is $100 \mathrm{~V}$ and the pressure in the vicinity of the extraction electrode is about $10^{-5}$ mbar (Ref. 10).

between 1810 and $1860{ }^{\circ} \mathrm{C}$, their intensity increase is higher than their vapor pressure variation, indicating a slight increase in ionization efficiency.

The ionization efficiency of IRENA feasibility prototype has been measured on ${ }^{84} \mathrm{Kr}$ using a calibrated leak of $9 \times 10^{-7} \mathrm{mbar} 1 / \mathrm{s}$, giving a value slightly higher than $0.3 \%$ for the cathode at $1860{ }^{\circ} \mathrm{C}$. In these conditions, the electron bombardment current is only $50 \mathrm{~mA}$. At such a low current, the radial bombarding electrons cannot properly confine the generated plasma. For EBGP, the ionization efficiency of $35 \%$ for $\mathrm{Kr}$ has been obtained for an electron current of $0.9 \mathrm{~A}$ and with a pressure in the ion source about two orders of magnitude lower.

Although measurements are still in progress to get exhaustive data in all the possible operation conditions, all these results already show main convergences with EBGP. Furthermore, the tests and Lorentz simulations carried out also give information for designing the next prototype. In particular, the mechanical reliability of anode fixation with regard to the cathode has to be improved.

\section{SELECTIVITY}

In addition to the fundamental operation specifications exposed, an essential exploitation specification has to be integrated: selectivity, i.e., nuclear beam purity. The purity of nuclear beams delivered by next generation facilities is a major concern in various aspects. First, a substantial part of nuclei of interest has very low production cross section, up to a few orders of magnitude lower than nuclei close to the stability valley. The exploitation of such beams of interest can only be considered in the absence of contamination by more commonly produced isotopes. Although beam purification can be carried out along the radioactive beam lines, e.g., by a high-resolution mass separator, it is of prime importance to be able to get the highest selectivity in the TIS itself. Such a high selectivity allows to keep the main part of unwanted 
generated radio nuclei confined in the TIS and to reduce the activation of the successive beam-line elements of the facility.

Even if FEBIAD-type ion sources are considered as nonselective, ${ }^{11}$ a proper design of the transfer line including selective trapping can make such sources as selective as any electron cyclotron resonance (ECR) or electron beam ion source (EBIS).

In contrast, surface ion source and resonant ionization by laser ion source (RILIS) which are considered as selective ion sources may have their selectivity and efficiency altered by the strong vapor flow.

For surface ion source, this may occur by surface contamination, depending on the nature of the elements released in the vapor flow.

For RILIS, if the pressure in the hot cavity is really high, then nonresonant ionized metals even those with higher potential ionization than the element of interest may create enough ions to deteriorate the plasma conditions in the cavity. ${ }^{12}$ In addition, the lack of electron emission from the cavity at high temperature will further degrade the plasma confinement.

\section{R\&D PERSPECTIVES}

A large number of tests and calculations are still required to work out an effective and operational IRENA prototype. However, the IRENA device already shows advantageous features. Its configuration is very close to RILIS hot cavity or thermoionization source. This makes IRENA a very adaptable complementary ion source. Furthermore, some IRENA developments should benefit both RILIS and thermoionization source developments. For instance, to prevent plasma deterioration in RILIS ionization cavity at high pressure operation, a remedy could consist in using IRENA radial structure (see Fig. 3) and applying a low arc voltage to generate lots of radial electrons at low energy only to confine the plasma. Tests are planned in a second step to determine the best conditions for providing such a confinement without inducing ionization so as to keep highest selectivity.

IRENA configuration also represents one of the most minimal configurations of discharge ion source, without magnetic confinement, so it is one of the most reliable candidates for operating in strong-radiation environment.

By developing a transfer tube with adjustable cooling and heating, IRENA would gain in selectivity with, in addition, the option of functioning in a thermoionization mode. In this way, this ion source gives the possibility of accessing at once a large variety of nuclear beams without the constraint of changing ion source.

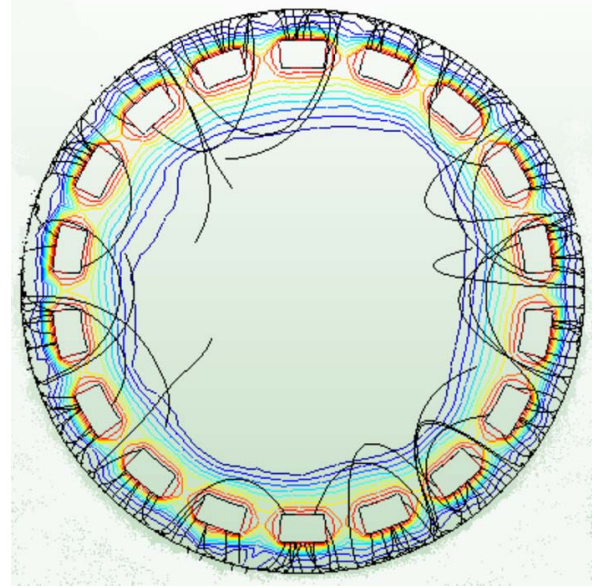

FIG. 3. (Color online) Simulation of electron emission in IRENA ionization chamber exploited in confining mode for RILIS. The anode volume plays the role of the cavity. Using Lorentz-EM code, the calculated electron trajectories have been obtained for $10 \mathrm{~V}$, taking into account space charge effect. From the simulation, electron density current of about $0.3 \mathrm{~A} / \mathrm{cm}^{2}$ can be obtained with such a low arc voltage.

\section{ACKNOWLEDGMENTS}

The authors acknowledge financial support of the EC under the PF6 "Research Infrastructure Action-Structuring the European Research Area" EURISOL-DS Project Contract No. 515768 RIDS. The EC is not liable for any use that may be made of the information contained herein.

\footnotetext{
${ }^{1}$ N. Lecesne, O. Bajeat, C. Eleon, S. Essabaa, C.-E. Demonchy, C. Feierstein, G. Gaubert, Y. Huguet, P. Jardin, C. Lau, F. Launay, F. Lemagnen, R. Leroy, J. Y. Pacquet, F. Pellemoine, A. Pichard, and L. Serani, Rev. Sci. Instrum. (these proceedings).

${ }^{2}$ Y. Blumenfeld, "Radioactive Beam Facilities in Europe: Status and Plans," Nucl. Instrum. Methods Phys. Res. B (to be published).

${ }^{3}$ For example, see A. Holmes-Siedle and L. Adams, Handbook of Radiation Effects (Oxford University Press, New York, 2002) M. Tavlet, A. Fontaine, and H. Schönbacher, CERN Yellow Report No. CERN 98-01, 1998.

${ }^{4}$ R. Kirchner, Nucl. Instrum. Methods Phys. Res. B 204, 179 (2003).

${ }^{5}$ C. Lau, M. Cheikh Mhamed, S. Essabaa, J. Arainer, P. Jardin, R. Leroy, C. Huet-Equilbec, O. Bajeat, and H. Lefort, Rev. Sci. Instrum. 77, 03 A706 (2006).

${ }^{6}$ M. Cheikh Mhamed, C. Lau, S. Essabaa, J. Arianer, and O. Bajeat, Rev. Sci. Instrum. 77, 03A702 (2006).

${ }^{7}$ J. Lesrel, J. Arianer, M. Arianer, O. Bajeat, J.-M. Buhour, H. Bzyl, F. Carrey, M. Chabot, J.-L. Coacolo, T. Corbin, J.-M. Curaudeau, F. Doizon, M. Ducourtieux, J.-M. Dufour, S. Essabaa, D. Grialou, C. Joly, M. Kaminski, H. Lefort, B. Lesellier, G. Magneney, L. Mottet, Y. Ollivier, C. Planat, M. Raynaud, Y. Richard, A. Said, A. Semsoum, F. Taquin, C. Vogel, G. Bienvenu, J.-N. Cayla, and M. Desmons, Proceedings of the European Particle Accelerator Conference "EPAC'06" (unpublished).

${ }^{8}$ J. M. Nitschke, Nucl. Instrum. Methods Phys. Res. A 236, 1 (1985).

${ }^{9}$ M. Cheikh Mhamed, C. Lau, and S. Essabaa, Rev. Sci. Instrum. (these proceedings).

${ }^{10}$ M. Cheikh Mhamed, Ph.D thesis, Université d'Evry Val d'Essonne, 2006.

${ }^{11}$ R. Kirchner, Nucl. Instrum. Methods Phys. Res. B 70, 186 (1992).

${ }^{12} \mathrm{~V}$. Fedoseev, personal communication (30 January 2007).
} 